\title{
Fault Identification on a Bearing Component of a Rotating Machine Using Vibration Spectrum Analysis
}

\author{
KT Aminu ${ }^{1}$, B D Halilu ${ }^{1}$, D Y Dabs ${ }^{1}$, M A Sule ${ }^{1}$ and S U Ibrahim ${ }^{2}$ \\ ${ }^{1}$ Department of Electrical Engineering, Abubakar Tatari Ali Polytechnic Bauchi, Nigeria \\ ${ }^{2}$ Department of Electrical Engineering, Federal Polytechnic Kaltungo, Gombe, Nigeria
}

\begin{abstract}
This paper presents an analysis of the vibration behavior of a rotating machine based on signal spectrum for detecting localized defects in rotating bearings. This approach can extract the characteristic frequencies related to the defect from the resonant frequency band of the vibration signal. The technique is demonstrated on a machine tool with ball bearings (bearing number "2" and bearing number "4") under simulated crack on the bearing cage. Experimental results show some preliminary evidence that the vibration spectrum technique can be used to locate and predict the failure of rotating machine rolling element bearings from vibration data.
\end{abstract}

Keywords: Vibration; Fault Identification; Bearings; Signal Spectrum; Condition Monitoring.

\section{INTRODUCTION}

Condition monitoring of the bearing component of a rotating machine provides advantages in the perspective of safety, operation and maintenance [ $1-10]$. A commonly used method is the vibration spectrum analysis. In this traditional approach, the key indicator of the fault is the characteristic defect frequencies in the vibration spectrum. The characteristic defect frequencies depend on the rotational speed and the location of the defect in a bearing [5]. The existence of one of the defect frequencies in the direct or processed frequency spectrum is strong indication of a fault in the system. The trace of the defected bearing is spread across a wide frequency band and can be easily masked with low frequency machinery vibrations and noise. The consecutive impacts between the defect and rolling elements triggers the resonances of the structure and the resonant frequencies dominate the frequency spectrum [5], [7]. Therefore, the characteristic defect frequencies cannot be easily traced as a result of their low amplitudes with respect to resonant amplitudes. A good review on the frequency domain analysis can be found in [10].

In practical applications, different techniques are used to amply the amplitudes at the characteristic defect frequencies. Most recently, the envelope method is one of them and frequently used in fault detection applications [4], [5]. The envelope technique can be viewed as digitizing the time signal and band-pass filtering it in a region, typically around the resonances of the structure due to their high signal-to-noise ratio. The difficulty in industrial applications is that resonances of the whole bearing structure cannot be easily predicted and consequently selection of the band-pass filter limits becomes difficult [5]. However, a new technique has been developed for the envelope analysis that involves ensemble empirical mode decomposition (EEMD) in the fault identification and detention of ball bearing [4].This approach recently developed by Hilbert-Huang Transformation (HHT) is applied to non-stationary phenomena. The EEMD is included in the so-called HHT based on the extraction of the energy associated with the intrinsic time scales in the signal. This process results in a set of components, called the intrinsic modes functions (IMF). The frequency components in each IMF not only relates to the sampling frequency, but also change with the signal itself. Furthermore, the Hilbert spectrum of each IMF will not lead to energy diffusion and leakage. Notwithstanding, however, the concept of envelope analysis has gone beyond scope of what this paper focused on, more information can be found in [4], [5], [8]. Further studies regarding the role of envelope analysis in bearing fault identification and detection would be worthwhile.

This paper has been organized as follows: section I, gives a brief introduction. Section II, gives a brief description of structural defect-induced vibration. Section III, presents bearing faults identification based on vibration spectrum. Section IV, gives the bearing defects and Section V, highlights the bearing failure stages. Section VI, presents the traces from the experimental campaigns. Section VII, gives the main conclusions derived from this paper. 
International Journal of Advances in Scientific Research and Engineering (ijasre), Vol 7 (9), September -2021

\section{STRUCTURAL DEFECT-INDUCED VIBRATION}

In this section, the form of a defect signal is briefly discussed. Motor pumps for instance, due to the rotating nature of their internal pieces (bearings), produce vibrations. In most cases, bearing failures are the result of material fatigue in the bearing [2]. During bearing operation, the sensing elements (accelerometers) strategically placed in points next to bearings and motors continuously allow the position, velocity or acceleration of the machine over time to be measured, thus generating a discrete signal of the vibration level. In general, the orientations of the sensors follow the three main axes of the machine, i.e. vertical, horizontal and axial. The major advantage of this technique over the previous efforts is the close vicinity of the fault detection mechanism to the fault source [6]. The pattern of the vibration signal consists in a successions of oscillations which repeat with each pass of a moving component over the fault. The repetition frequency of the impact depends on the position of the fault. The fault can be on the inner race, the outer race or on the rolling element [2].

\section{BEARING FAULTS IDENTIFICATION BASED ON VIBRATION SPECTRUM}

Whenever a faults occurs on a bearing component, impacts are generated in operation, which in turn excite the bearing and its support structures. The resulting resonance signatures also known as the characteristics defect frequencies are usually amplitude modulated by the bearing defect, therefore the analysis of these resonance signatures plays a key role in vibration-based bearing fault identification and detection [8]. The spectrum analysis takes the incoming signal and breaks it into its individual frequencies by using either analogue filters or a software process called Fourier analysis (analogous to the human ear selecting the noise of individual instruments within an orchestra). This process is extremely powerful and is used extensively for trending and diagnosis; it relies on the ability to link particular frequencies to particular component defects, such as bearings or gears. However, spectra generate large volumes of information which require expert staff or software to interpret them. Indeed, the existence of the characteristic defect frequencies in the vibration spectrum of the signal indicates a defect [5].

\section{BEARING DEFECTS}

A bearing defect can produce different characteristic fault frequencies in the vibration spectrum of the rotating machine. These frequencies are predictable and defend on the surface of the bearing which contains the fault [2]. The bearing defects cause periodic impulses in vibration signals. Amplitude and period of these impulses are determined by shaft rotational speed, fault location and bearing dimensions. Therefore a specific frequency can be attributed to each component of the bearing [2]. The fundamental cage frequency is given by:

$$
f_{c}=\frac{f_{r}}{2}\left(1-\frac{d}{D} \cos (\alpha)\right)
$$

The ball defect, respectively the inner race defect frequencies can be computed by using the following equations:

$$
\begin{gathered}
f_{b d}=\frac{D}{d} f_{r}\left(1-\frac{d^{2}}{D^{2}} \cos ^{2}(\alpha)\right) \\
f_{i d}=n\left(f_{r}-f_{c}\right)=\frac{n f_{r}}{2}\left(1-\frac{d}{D} \cos (\alpha)\right)
\end{gathered}
$$

The formula for computing the outer race defect frequencies is the following:

$$
f_{o d}=n f_{c}=\frac{n f_{r}}{2 d}\left(1-\frac{d}{D} \cos (\alpha)\right)
$$

where, $f_{r}$ is the rotor speed, $n$ the number of balls, $d$ the diameter of the ball, $D$ the pitch diameter of the bearing and $\alpha$ is the contact angle. The typical value of the contact angle is 0o. For most bearing with six to twelve balls, the frequencies given by (3) and (4) can be approximated with [2]:

$$
\begin{aligned}
& f_{i d}=0.6 \cdot n \cdot f_{r} \\
& f_{\text {od }}=0.4 \cdot n \cdot f_{r}
\end{aligned}
$$

It should be noted that any air gap eccentricity produces anomalies in the air-gap flux density which is reflected on the stator current. In the case of the bearing fault the characteristic fault frequencies are modulated by the electrical supply frequency at a predictable frequency [2]. 
International Journal of Advances in Scientific Research and Engineering (ijasre), Vol 7 (9), September -2021

$$
f_{\text {bng }}=\left|f_{s} \pm m \cdot f_{V}\right|
$$

where $f_{s}$ is the electrical supply frequency, $f_{V}$ one of the fault frequencies defined by Equations (1) - (4) and $m=1,2,3 \ldots$

\section{BEARING FAILURE STAGE}

During the failure stage, the bearing develops flaws that are visible to the human eye. At this stage, the bearing usually produces audible sound, and the temperature of the bearing will rise. Vibration amplitudes in the "bearing-related" range (prime spike) reach easily detectable levels. Once the failure stage is reached, it is necessary to either change the bearing or increase the frequency of monitoring to estimate how long the bearing will safely operate before causing a catastrophic machine failure. This stage is considered the economical time at which to replace the bearing. If the bearing is not removed during the failure stage, it will eventually enter the final progression of failure, the catastrophic stage.

When the bearing enters this stage, rapid failure of the bearing is imminent. Audible noise produced by the bearing increases significantly, and the bearing temperature increases until the bearing overheats. Rapid wear causes the bearing clearance to increase, which then allows significant shaft motion relative to the bearing. Since a rolling element bearing is designed to restrict shaft motion, it can be very dangerous to allow the bearing to reach this stage due to the probability of creating a rub within the machine. Bearing-related (prime spike) vibration amplitude levels will show significant increases in this stage. High frequency vibration data may be unreliable in this stage and caution should be used in its interpretation. Due to "self-peening" of the bearing flaws, high frequency amplitude levels often decrease during this stage, and it can appear that the bearing is in an earlier stage of failure. The occurrence of this "self-peening" phenomenon is especially true for low speed machines.

\section{EXPERIMENTAL TRACES}

Vibration data from two bearings numbered "2" and "4" was used in the analysis. The vibration data was acquired at a spindle speed of $1800 \mathrm{rpm}$ corresponding to a rotating frequency $f_{r}$ of $30 \mathrm{~Hz}$. The ball diameter to pitch diameter ratio $\frac{d}{D}$ for the bearings is 0.22 and the contact angle $\alpha=0^{\circ}$. For bearing number "2", there are 11 balls in the bearing. Therefore according to Equations (1), (2), (3) and (4), the characteristic frequency of the cage defect, ball defect, inner race defect and the outer race defect are computed to be $11.7 \mathrm{~Hz}, 201.3 \mathrm{~Hz}, 128.7 \mathrm{~Hz}$ and $129.7 \mathrm{~Hz}$ respectively.

The original vibration signal of bearing number "2" is depicted in Figure 1. It is clear that there are periodic impacts in the vibration signal. There are significant fluctuations in the peak amplitude of the signal, and there are also considerable variations of frequency content. From Figure 1, we can hardly find any of the characteristic period of the defects.

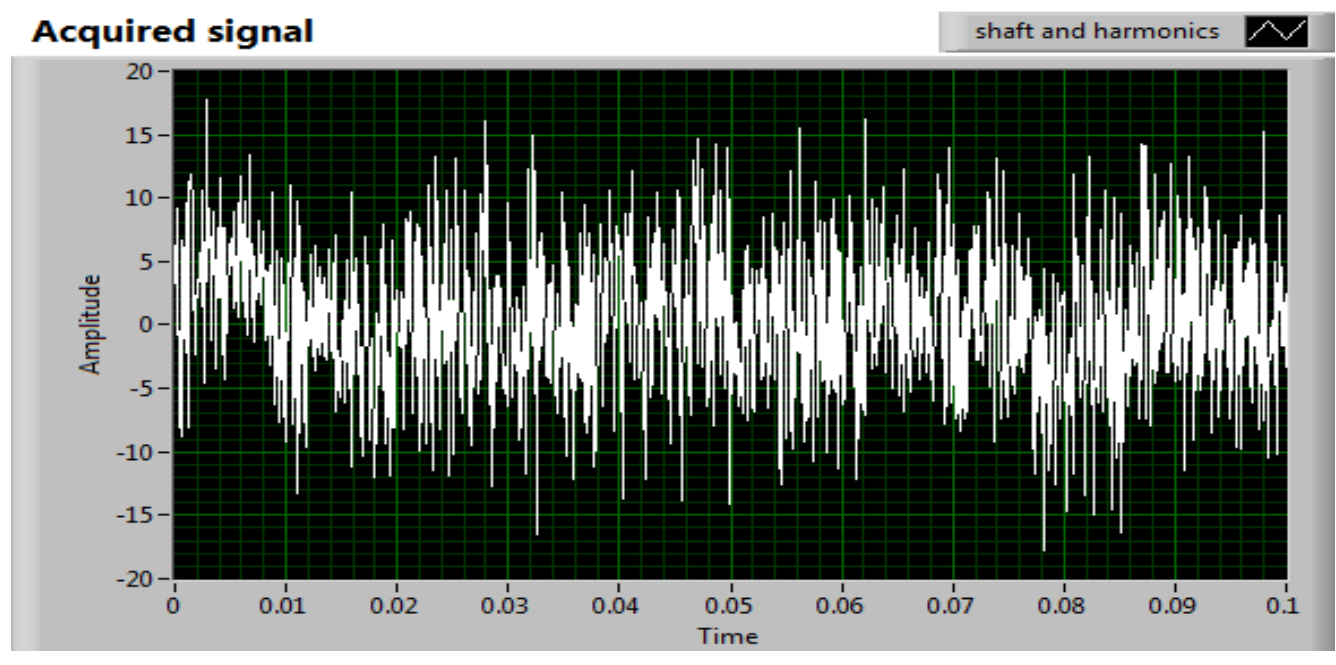

Figure 1. Vibration signal for bearing number "2"

Figure 2 displays the power spectrum of the vibration signal for bearing number " 2 ". The resulting vibration power spectrum shown in Figure 2 is a series of pulses occurring at integer multiples of $f_{c}$. The main harmonics of the spectrum are located at $n f_{c}(n=0, \pm 1, \pm 2, \ldots)$. Evidently, Figure 2 presents an identified, predicted, theoretical vibration spectrum for ball bearings within the specified range, each having a cage defect. 
International Journal of Advances in Scientific Research and Engineering (ijasre), Vol 7 (9), September -2021

\section{power spectral density}

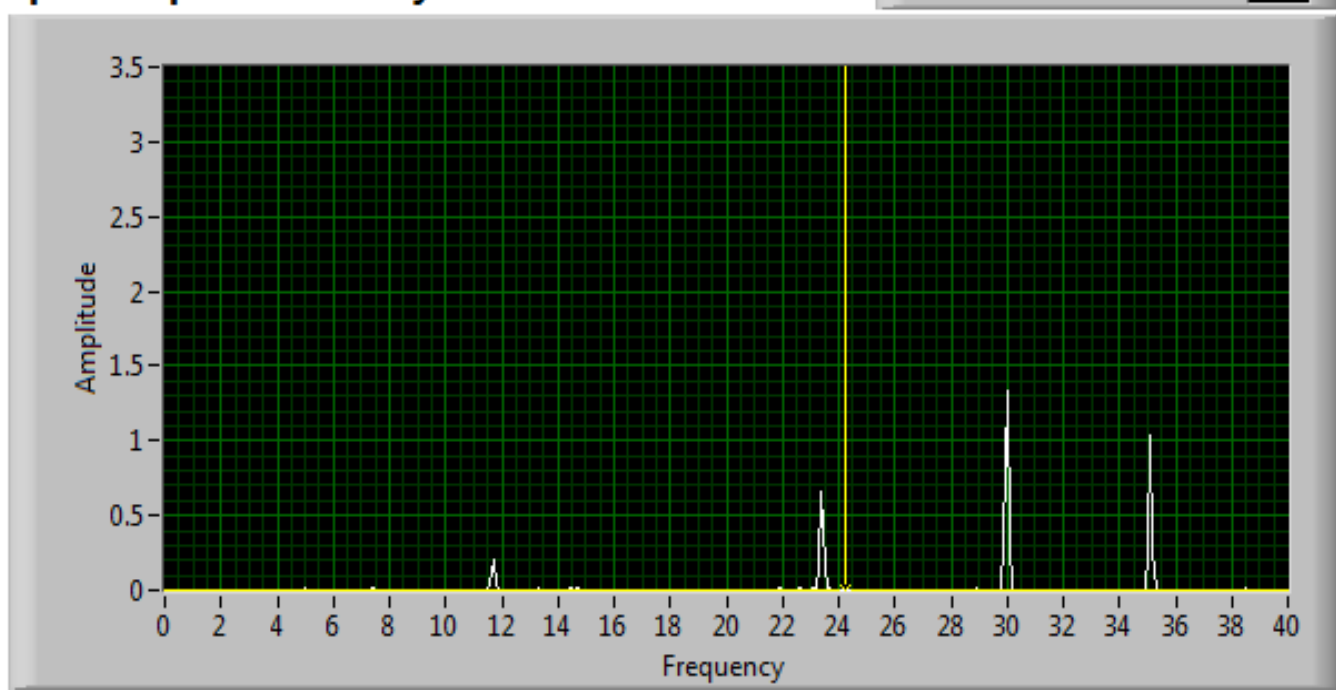

Figure 2. Power spectrum of the vibration signal for bearing number " 2 " emphasizing bearing cage defect

Similarly, for bearing number "4" with 9 balls and evaluating Equations (1), (2), (3), and (4) with the given parameters, the characteristic frequencies of the cage defect, ball defect, inner defect and outer defect are $11.1 \mathrm{~Hz}, 107.6 \mathrm{~Hz}, 170.1 \mathrm{~Hz}$ and 99.9 $\mathrm{Hz}$ respectively. These fault frequencies appear when the bearing is defective, and therefore it is possible to identify the source of the bearing problem by observing the spectrum of the vibration signal. The acceleration signal generated by a bearing number " 4 " is shown in Figure 3.

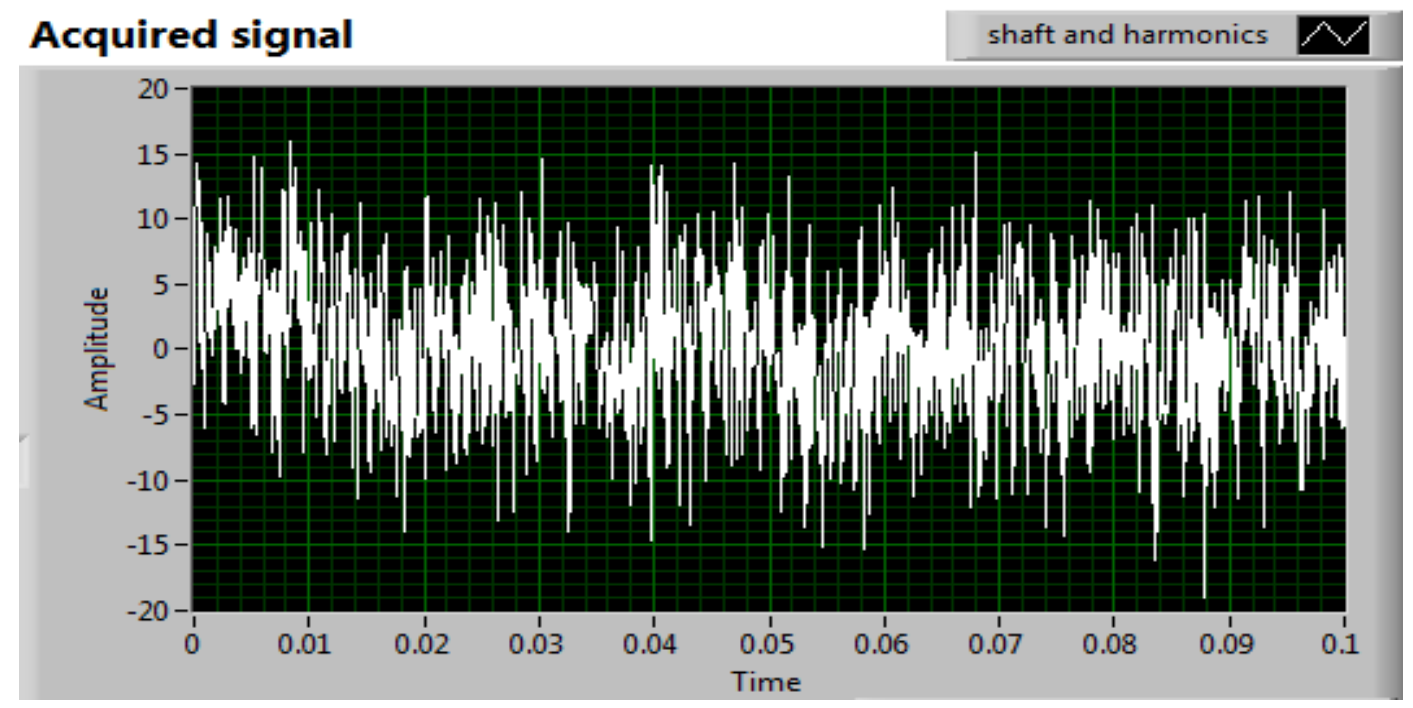

Figure 3. Acceleration signal generated by bearing number "4"

It seen that the periodic features, carried by the vibration signal in Figure 3, can be clearly recognized. These periodic features are equally spaced and repeated at approximately constant rate. Having visualized raw vibration signal characteristics, the next step is to examine characteristic defect frequencies (i.e cage defect, ball defect, inner defect and outer defect) from the vibration spectrum. Figure 4 depicts the vibration spectrum for bearing number " 4 ". 


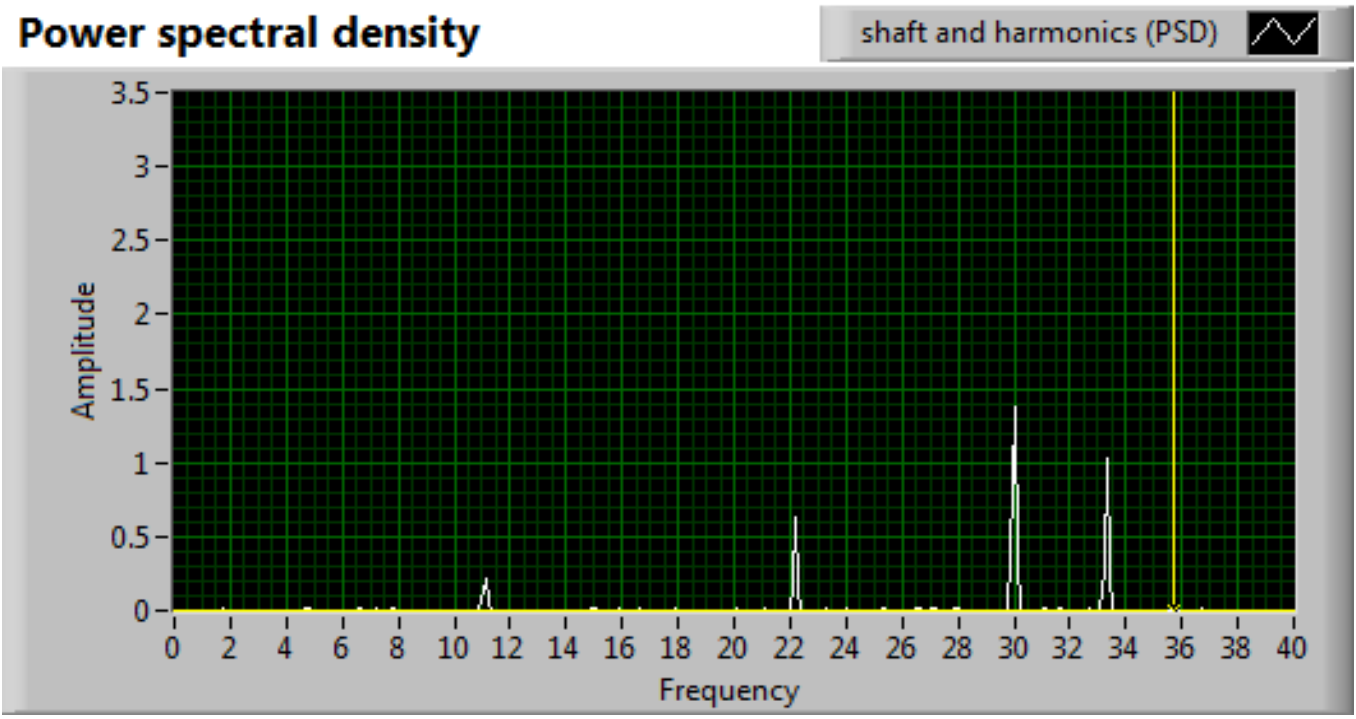

Figure 4. Vibration spectrum for bearing number "4" fault identification and detection

Bearing health conditions are estimated by analyzing the related characteristic frequency component (i.e $f_{c}, f_{b d}, f_{i d}$, and $f_{o d}$ ) in the resulting spectra. Figure 4 demonstrates the resulting spectra determined by the frequency domain analysis technique on the vibration signal shown in Figure 3. It seen that the defect frequency (approximately $11.1 \mathrm{~Hz}$ ) can be clearly recognized; in this case, the defect occurs on the bearing's cage, that is $f_{c}=11.1 \mathrm{~Hz}$ when the spindle speed is $30 \mathrm{~Hz}$.

\section{CONCLUSION}

Condition monitoring and fault identification and diagnosis have received an important measure for predictive maintenance and condition based maintenance of bearing element of rotating machinery operation. Although many methods existing in the industries can be directly or indirectly applied, bearings of rotating machine present a particular challenges for successful and reliable diagnostics and prognostics. In this paper signal processing and vibration spectrum analysis techniques were employed to classify faults in bearing components of rotating machines. The spectrum analysis provides the feature vector used in the subsequent classification steps. Based on this comprehensive investigation, it is found that it is usually difficult to clearly recognize characteristic defect frequencies using this approach, because sometimes it is difficult to clearly see the fundamental defect frequency even though their harmonics were evident. It should however be noted that, high frequency measurements are to be used only as a possible indicator of impending rolling element bearing failure and should generally not be used as the primary indicator to determine when to replace the bearing.

\section{ACKNOLEDGEMENTS}

The authors would like to thank the reviewers for their valuable contributions.

\section{REFERENCES}

1. Zhang, L., and Lang, Z. 2018. Wavelet Energy Transmissibility Function and its Application to Wind Turbine Bearing Condition Monitoring, IEEE Transactions on Sustainable Energy, Vol. 9 (4). Pp. 1833 - 1843

2. Harlisca, C., and Szabo, L. 2012. Bearing Faults Condition Monitoring - A Literature Survey, Journal of Computer Science and Control Systems, Vol. 5 (2). Pp. $19-22$

3. Janjarasjitt, S., Ocak, H., and Loparo, K. A. 2008. Bearing Condition Diagnosis and Prognosis Using Applied Nonlinear Dynamical Analysis of Machine Vibrational Signal, Journal of Sound and Vibration, 317. Pp. 112 - 126

4. Ai, S., and Yuping Zhang, H. 2009. Condition Monitoring for Bearing Using Envelope Spectrum of EEMD, In proceedings of International Conference on Measuring Technology and Mechatronics Automation. Pp. 190 - 193

5. Kiral, Z., and Karagulle, H. 2003. Simulation and Analysis of Vibration Signals Generated by Rolling Element bearing with Defects, Tribology International, 36. Pp. $667-678$

6. Holm-Hasen, T. B., and Gao, X. R. 2000. Vibration Analysis of a Sensor-Integrated Ball Bearing, Transactions of the ASME, Journal of Vibration and Acoustics, Vol. 122. Pp. $384-392$

7. Kulkarni, S., and Bewoor, A. 2006. Vibration Based Condition Assessment of Ball Bearing with Distributed Defects, Journal of Measurements in Engineering, Vol. 4 (2). Pp. 87 - 94 
8. Liu, J., Wang, W., and Ma, F. 2011. Bearing System Health Condition Monitoring Using Wavelet Cross-Spectrum Analysis Technique, Journal of Vibration and Control, Vol. 0 (0). Pp. 1 - 11

9. Halme, J., and Anderson, P. 2009. Rolling Contact Fatique and Wear Fundamentals for Rolling Bearing Diagnostics State of the Art, Review Paper. Pp. 377 - 393

10. KiranKumar, M. V., Lokesha, M., Kumar, S., and Kumar, A. 2018. Review on Condition Monitoring of Bearings Using Vibration Analysis Techniques, IOP Conference Series: Materials Science and Engineering, 376. Pp. 1 - 6 